\title{
Effect of guanethidine on the natural history of post-traumatic algodystrophy
}

\author{
J Field, R M Atkins
}

\begin{abstract}
Objectives-To determine the effect of intravenous regional guanethidine on post-traumatic algodystrophy.

Methods-Ten of 20 consecutive patients with algodystrophy after Colles' fracture were treated with serial intravenous regional guanethidine blockades and the other 10 were treated with physiotherapy alone. Patients were assessed before and after each block and then monthly over a six month period.

Results-The treated patients had subjective and objective improvements in the assessed features of algodystrophy; treatment resulted in a significant reduction in finger tenderness at five and six months. Conclusions-Guanethidine blockade induces short term benefits in the symptoms of algodystrophy but improves finger tenderness only in the long term.
\end{abstract}

(Ann Rheum Dis 1993; 52: 467-469)

Algodystrophy (reflex sympathetic dystrophy or Südeck's atrophy) is a syndrome affecting the hands and feet and occurring most often after trauma. ${ }^{1}$ In the hands the disorder consists of excessive tenderness of the fingers, joint stiffness, swelling, and vasomotor instability (temperature and sudomotor control). As the disorder progresses regional osteoporosis and atrophy of the subcutaneous tissues occur, leading to debilitating soft tissue contractures.

Hannington-Kiff first reported the technique of intravenous regional guanethidine for the treatment of algodystrophy ${ }^{2}$ and since then its use has been widely accepted, though Casale et $a l^{3}$ have noted the effectiveness of the tourniquet only. Studies have mainly relied on subjective evidence of improvement using this treatment, ${ }^{45}$ but objective evidence of short term improvement after guanethidine treatment has been reported. ${ }^{6}$

It is the purpose of this paper to assess the benefit of guanethidine on the quantifiable objective measurement of algodystrophy and to determine its effect on the natural history of of Orthopaedics, Bristol Royal Infirmary, Bristol BS2 8HW, United Kingdom J Field

R M Atkins

Correspondence to: Mr Field.

Accepted for publication 11 February 1993
From March to August 1991 all patients with Colles' fracture were assessed at nine weeks after fracture for evidence of algodystrophy. Twenty patients fulfilled the criteria for diagnosis, namely an abnormal dolorimetery ratio, considerable finger swelling and stiffness, and the presence of vasomotor instability. The first 10 were treated by serial guanethidine blockade until clinical improvement. At this time reports that guanethidine blockade might be no better than transient inflation of a tourniquet prompted us to review our treatment policy (Casale $e t a l^{3}$ ) and hence the next 10 patients were not offered guanethidine blockade. In every other aspect treatment of the two groups was identical. Fortuitously, this gave us an opportunity to investigate the response to guanethidine blockade.

After fracture the patients were treated in a plaster cast for five weeks, all fractures having been manipulated under intravenous regional analgesia.

ASSESSMENT METHODS

Subjective

After each guanethidine block the patients were asked to assess improvement in each of their symptoms using a visual analogue scale. ${ }^{7}$

\section{Objective}

Assessments of features of algodystrophy were made using techniques described in detail elsewhere ${ }^{8}$ and reproducibility studies were performed in 20 control patients.

(1) Finger tenderness. Using a dolorimeter, a ratio of abnormal/normal hands was obtained. The $95 \%$ lower confidence limits of the dolorimetry ratio in normal subjects of this age is 0.90 . The reproducibility of the method was $2 \cdot 28 \%$.

(2) Swelling. Circumference of the fingers was measured using an arthrocircameter. ${ }^{9}$ The normal range was $-25 \mathrm{~mm}$ to $+25 \mathrm{~mm}$. The reproducibility of method was $7 \cdot 19 \%$.

(3) Finger stiffness. A total range of movement of the finger joints of each hand was obtained using a goniometer. The value for joint stiffness was found by subtracting the total for one hand from the other (normal range $-63^{\circ}$ to $+63^{\circ}$; reproducibility was $1 \cdot 47 \%)$.

(4) Vasomotor instability. This was assessed by a questionnaire. ${ }^{8}$ Each feature was scored as 0 (absent) or 1 (present) and the sum of these scores was used to give a semiquantitative assessment of vasomotor instability.

(5) Grip strength. This was measured using a Jamar dynamometer, ${ }^{10}$ and the value expressed as a ratio of the normal hand (normal range $0 \cdot 75-1 \cdot 27$ ). 
Patients in each group were assessed at nine weeks, at which time the diagnosis was made. The mean time from fracture to the start of treatment was three months (range two to four months), the timing of treatment depending on clinical severity. In each patient treated there was a transient vasodilatory response indicating successful sympathetic interruption.

In the treated group, subjective assessments

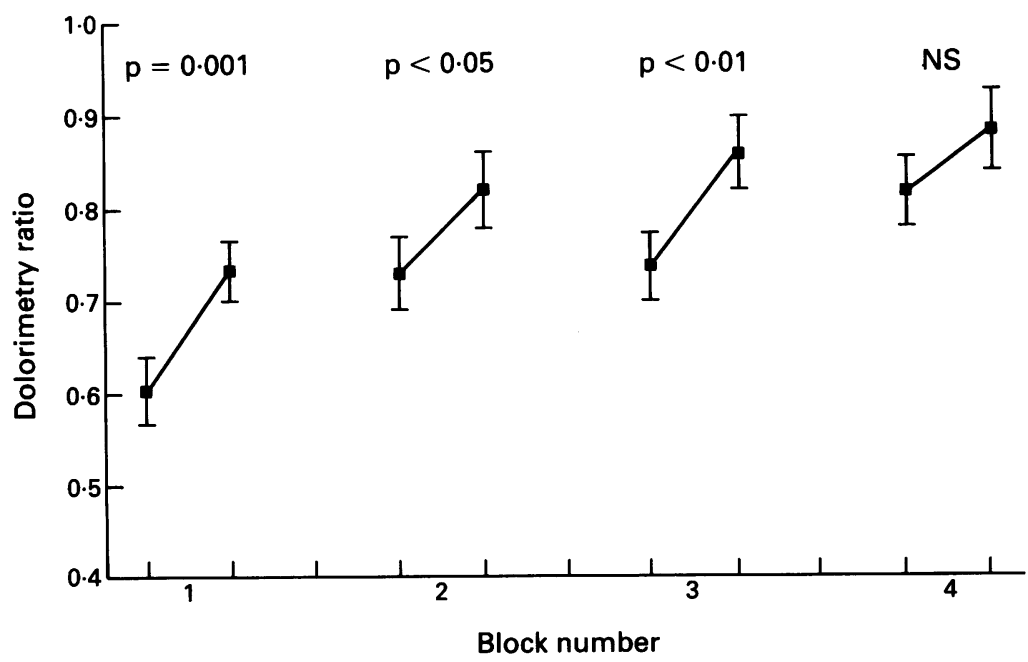

Figure 1 Improvements in finger tenderness measured by dolorimetry ratio before and after each guanethidine block (mean (SEM)).

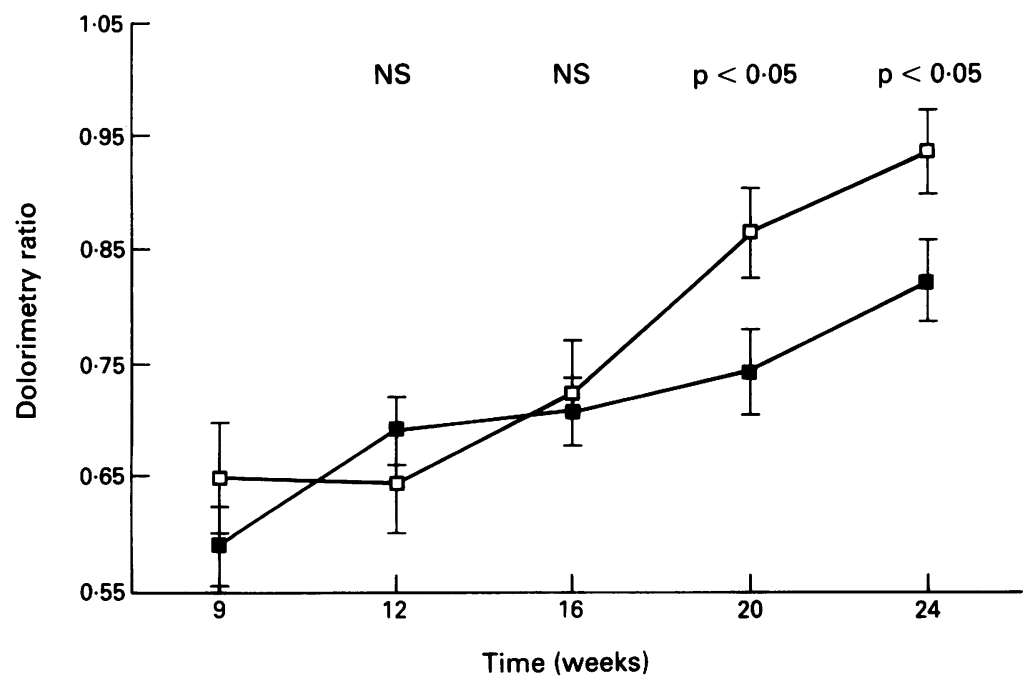

Figure 2 Effect of guanethidine treatment on finger tenderness measured by dolorimetry ratio over a six month period. Treated group $(\square)(n=10)$; untreated group $(\square)(n=10)$.

Mean values of each parameter before and after treatment in those treated with guanethidine blockade

\begin{tabular}{lccl}
\hline Parameter & Mean before & Mean after & Significance \\
\hline Block 1 & & & \\
Finger stiffness (degrees) & 361.3 & $182 \cdot 2$ & $\mathrm{p}<0.0001$ \\
Finger swelling (cm) & 20.9 & 5.3 & $\mathrm{p}<0.05$ \\
Grip strength (kgf) & 0.12 & 0.37 & $\mathrm{p}=0.01$ \\
Block 2 & 282 & 240.5 & $\mathrm{NS}$ \\
Finger stiffness (degrees) & 16.7 & $12 \cdot 2$ & $\mathrm{NS}$ \\
Finger swelling (cm) & 0.19 & 0.26 & $\mathrm{p}<0.05$ \\
Grip strength (kgf) & 273.1 & $230 \cdot 2$ & $\mathrm{NS}$ \\
Block 3 & 15.86 & 14 & $\mathrm{NS}$ \\
Finger stiffness (degrees) & 0.21 & 0.34 & $\mathrm{p}<0.05$ \\
Finger swelling (cm) & 268 & 182.2 & $\mathrm{p}<0.05$ \\
Grip strength (kgf) & 12.83 & 5.33 & $\mathrm{NS}$ \\
Block 4 & 0.29 & 0.37 & $\mathrm{p}<0.05$ \\
Finger stiffness (degrees) & & & \\
Finger swelling (cm) & & & \\
Grip strength (kgf) & & &
\end{tabular}

of the change in symptoms using visual analogue scales were made after each block. ${ }^{7}$ Patients were assessed objectively before and between 24 and 48 hours after each block.

After treatment all patients were reviewed monthly for six months; guanethidine blockade was repeated if the patient had sufficient subjective symptoms up to a maximum of four blocks. Ten patients received two blocks, eight received three blocks, and six received four blocks.

\section{STATISTICAL ANALYSIS}

The significance of differences between means was assessed using Student's $t$ test. This study was approved by the local ethical committee.

\section{Results}

There was no significant difference between the treated and untreated group in age, sex, Frykman $^{11}$ and Lidstrom ${ }^{12}$ fracture type (the former taking into account the ulnar styloid, the latter fracture displacement), number of manipulations, or adequacy of reduction.

SUBJECTIVE IMPROVEMENT

Every patient had a subjective improvement of symptoms. The average final subjective improvement was $78 \cdot 5 \%$ (range $10-100 \%$ ).

\section{OBJECTIVE IMPROVEMENT}

Finger tenderness (fig 1; table) improved significantly after the first three blocks, though there was a gradual relapse between blocks. The dolorimetry ratio improved with time in the two groups, but the treated group had significantly fewer tender fingers at five and six months $(p<0.05)$.

Swelling improved significantly after the first and last block (table). The treated group did not resolve more quickly over the six month period.

Finger stiffness decreased significantly after the first and fourth block; the decrease over the longer period was not significant (table). The normal range was not reached by the examination at six months in either group.

Vasomotor instability was not different in the two groups.

Grip strength increased significantly with each block and time in the two groups, but the treated group fared no better (table).

\section{Discussion}

In the past the assessments of the treatment of algodystrophy have been made more difficult by the lack of homogeneity of patients and the lack of objective and quantifiable criteria for diagnosis and the assessment of the severity of the disorder. It has been previously shown that the diagnosis of a mild form of the syndrome occurs after Colles' fracture. ${ }^{8}$

Guanethidine blockade has been a recognised treatment of algodystrophy for some time, but until now its efficacy in 
relieving the physical abnormalities of the disorder as opposed to altering the sympathetic outflow to the limb has been mainly subjective. ${ }^{5}$

The treated group achieved short term objective improvements in finger tenderness, finger stiffness, swelling, and grip strength and these correlated with the patients' subjective impression of improvement, suggesting that the methods used are indeed assessing the disability which the patients experience from the syndrome.

In contrast, the effect on the natural history is less clear. It has been reported that the symptoms of algodystrophy improve with time after Colles' fracture. ${ }^{13}$ These results confirm this improvement but also show that guanethidine blockade administered under this regimen resulted in a significant improvement in pain only in the long term.

Four blocks have been recommended as the maximum before more permanent sympathetic blockade is considered. ${ }^{14}$ As all the patients treated obtained symptomatic benefit with four or less blocks, it was difficult to justify giving more; however, further blocks may have given more improvement and it may be argued that all patients should have received the same number of blocks.

There are a number of flaws in this study. The patients were not randomised, though they were well matched and the numbers studied were small. The changes in objective features in the short term and the finding of a significant improvement in finger tenderness over the longer period, however, suggests that this approach may be useful for further investigations of the efficacy of guanethidine blockade in the treatment of algodystrophy.

1 Doury P, Dirheimer Y, Pattin S. Algodystrophy: diagnosis and therapy of a frequent disease of the locomotor apparatus, $T$ Berlin: Springer, 1981 .

2 Hannington-Kiff J G. Pharmacological target blocks in hand surgery and rehabilitation. $f$ Hand Surg $[\mathrm{Br}]$ 1974; 9 $29-36$.

3 Casale R, Glynn C J, Buonocore $M$. The role of ischaemia in the analgesia which follows Bier's block technique. Pain 1992; 50: 169-75

4 Woosley R L. Nies A S. Guanethidine. N Engl f Med 1976; 295: 1053-7.

5 Glynn C J, Basedow R W, Walsh J A. Pain relief following post-ganglionic sympathetic blockade with I.V. guanethidine. Br $\mathcal{f}$ Anaesth 1981; 53: 1297-301.

6 Field J, Monk C, Atkins R M. Objective changes in algodystrophy following treatment with intravenous algodystrophy following treatment with in

7 regional guanethidine. F Hand Surg [Br] In press. 1127-31.

8 Atkins $R$ M, Duckworth $T$, Kanis J A. Features of algodystrophy after Colles' fracture. $\mathcal{F}$ Hand Surg $[\mathrm{Br}]$ 1989; 14: $161-4$.

9 Willkens R F, Gleichert J E, Gade E T. Proximal interphalangeal joint measurement by arthrocircameter. Ann Rheum Dis 1973; 32: 585-6.

10 Bechtol C O. Grip test: the use of dynamometer with adjustable hand spacings. $f$ Bone foint Surg [Am] 1954; 36: $820-4$.

11 Frykman G. Fracture of the distal radius and its complications including the shoulder, hand, finger syndrome, disturbance in the distal radio-ulnar joint and the impairment of ner. experimental study. Acta Orthop Scand Suppl 1967; 108.

12 Lidstrom A. Fractures of the distal end of the radius. A clinical and statistical study of end results. Acta Orthop Scand Suppl 1959; 41

13 Bickerstaff D R. Post traumatic algodystrophy [MD thesis] Sheffield: Sheffield Univ., 1990.

14 Schutzer S F, Gossling H R. The treatment of reflex sympathetic dystrophy syndrome. $\mathcal{f}$ Bone foint Surg $[\mathrm{Am}]$ 1984; 66: 625-9. 